\title{
TOP-ARBEITGEBER - WER SIND DIE BESTEN?
}

\subsection{Trendence Graduate Barometer Deutschland}

Seit 1999 führt das Berliner Trendence Institut jährlich die Studie Trendence Graduate Barometer (ehemals Absolventenbarometer) durch. Bei der Untersuchung werden abschlussnahe Studierende und Absolventen zum Berufseinstieg gefragt, welche potentiellen Arbeitgeber attraktiv sind und nach welchen Kriterien sie die erste Station im Berufsleben auswählen.

An den Befragungen im September 2013 und im Februar 2014 nahmen abschlussnahe Studierende aus wirtschaftswissenschaftlichen Fächern von 109 Hochschulen teil, 14.000 Antworten wurden ausgewertet. Dabei überraschen einige Befunde. So konnten etwa Lufthansa, die ProSiebenSAT1. Media AG, die Deutsche Bahn und Bayer sich auffällig verbessern. Zu den Verlierern 2014 gehören Amazon, Apple, aber auch IKEA und die Sparkassen. Bei einer Sicht auf geschlechtsspezifische Bewertungen fällt auf: Beide Geschlechter favorisieren Autohersteller wie Audi, BMW und VW. Für Männer ist die Lufthansa wenig attraktiv, für Frauen PwC. Google liegt bei Männern auf Rang 5, bei Frauen nur auf Platz 8. Männer sind offenbar sportlicher: Adidas rangiert bei ihnen auf Platz 5, bei Frauen erst auf 10 .

Top-Arbeitgeber 2014

\begin{tabular}{ccl} 
Rang & $\%$ & Unternehmen \\
\hline 1 & 12,5 & BMW Group \\
\hline 2 & 12,3 & Audi \\
\hline 3 & 8,6 & Volkswagen \\
\hline 4 & 7,5 & Lufthansa \\
\hline 5 & 7,4 & Porsche AG \\
\hline 6 & 6,5 & Daimler/Mercedes Benz \\
\hline 7 & 6,4 & Google
\end{tabular}


Top-Arbeitgeber 2014

\begin{tabular}{lll} 
Rang & $\%$ & Unternehmen \\
\hline 8 & 6,3 & Bosch Gruppe \\
\hline 9 & 6,0 & adidas AG \\
\hline 10 & 5,6 & Siemens \\
\hline 11 & 4,6 & PwC \\
\hline 12 & 4,3 & Ernst \& Young (EY) \\
\hline 13 & 3,8 & Auswärtiges Amt \\
\hline 13 & 3,8 & BASF \\
\hline 13 & 3,8 & KPMG \\
\hline 16 & 3,6 & Deutsche Bank AG \\
\hline 17 & 3,5 & Apple \\
\hline 18 & 3,4 & McKinsey \& Company \\
\hline 19 & 3,1 & Unilever \\
\hline 20 & 3,0 & BCG The Boston Consulting Group \\
\hline
\end{tabular}

Quellen: trendence Graduate Barometer 2014, www.trendence.com, http://www.deutschlands 100.de

\subsection{Arbeitgeberranking von WirtschaftsWoche und Universum}

Welches Unternehmen kann beim Recruiting auf hohe Akzeptanz bei kommenden „High Potentials“ setzen? Zwischen Juni und Oktober 2013 hat die Beratungsgesellschaft Universum Communications aus Köln 4.995 Ingenieure, Ökonomen, Informatiker und Naturwissenschaftler nach ihren bevorzugten Arbeitgebern gefragt. Die Teilnehmer waren unter 40, hatten zwischen 1 und 8 Jahren Berufserfahrung und wählten aus 150 Arbeitgebern bis zu 5 Favoriten aus. Auch freie Nennungen waren möglich.

Bei dem Arbeitgeberranking zeigt sich, dass junge Wirtschaftswissenschaftler gleiche Präferenzen haben wie Ingenieurwissenschaftler. Für beide sind die attraktivsten Arbeitgeber in Deutschland Audi, BMW und Porsche. Die Automobilbranche zieht Young Professionals mit Abstand am stärksten an. Überraschend hier: der Aufstieg von Ikea. Das Möbelhaus klettert zum Vorjahr um 13 Plätze auf Rang 9 nach oben. Das E-Commerce Unternehmen Amazon findet sich zum ersten Mal in den Top 20.

Die Berater von Universum registrieren dagegen, dass „Wirtschaftsprüfer und Unternehmensberater weiter Gegenwind“ spüren. Auch gegenüber Banken sind junge Berufstätige mit wirtschaftswissenschaftlichem Hintergrund reservierter als etwa gegenüber Traditionsunternehmen wie Bosch oder Siemens. Die Versicherungen konnten ihre Attraktivität etwas steigern. Weiterhin beliebt sind die Unternehmen der Konsumgüterindustrie. 
Universum fragte in der Professional Studie 2013 nicht nur nach den attraktivsten Arbeitgebern. Sie lotete auch Karriereziele, Jobzufriedenheit oder Wechselbereitschaft aus. Universum: „Der ideale Arbeitgeber sollte demnach ein attraktives Grundgehalt, ein freundliches Arbeitsumfeld, Anerkennung und eine sichere Anstellung bieten. Wichtigstes Karriereziel ist für die Young Professionals ein ausgewogenes Verhältnis zwischen Arbeit und Privatem, gefolgt von Jobsicherheit und der intellektuellen Herausforderung. “

Platzierung 2013

\begin{tabular}{lll}
\hline \multicolumn{1}{c}{ Rang } & $\%$ & Vorjahresrang \\
\hline 1 Audi & 14,3 & 2 \\
\hline 2 BMW Group & 13,1 & 1 \\
\hline 3 Porsche & 11,2 & 3 \\
\hline 4 Deutsche Lufthansa & 9,1 & 4 \\
\hline 5 Volkswagen & 8,9 & 6 \\
\hline 6 Google & 8,8 & 4 \\
\hline 7 Daimler/Mercedes-Benz & 7,1 & 9 \\
\hline 8 Siemens & 7,0 & 22 \\
\hline 9 IKEA Deutschland & 6,3 & 8 \\
\hline 10 adidas Group & 6,3 & 13 \\
\hline 11 Auswärtiges Amt & 6,1 & 11 \\
\hline 12 Robert Bosch & 5,9 & 10 \\
\hline 13 McKinsey \& Company & 5,4 & 13 \\
\hline 13 Nestlé Deutschland & 5,4 & 16 \\
\hline 15 Deutsche Bank & 5,2 & 18 \\
\hline 16 Hugo Boss & 5,2 & 28 \\
\hline 17 l'Oréal Deutschland & 5,1 & 19 \\
\hline 18 Europäische Zentralbank (EZB) & 5,0 & 20 \\
\hline 19 SAP & 4,7 & 7 \\
\hline 20 Amazon & & 7 \\
\hline
\end{tabular}

Quelle: www.wiwo.de/erfolg/jobsuche, Stand: Dez. 2013 


\subsection{Great Place to Work}

Das Institut Great Place to Work ${ }^{\circledR}$ begann 1981 in New York, Arbeitgeber aus Sicht der Mitarbeiter zu bewerten und auszuzeichnen. Great Place to Work ${ }^{\circledR}$ Deutschland wurde anlässlich des durch die EU-Kommission initiierten Wettbewerbs „Beste Arbeitgeber der EU 2003“ im Jahr 2002 in Deutschland gegründet.

Auf Basis von Benchmark-Untersuchungen zu Qualität und Attraktivität der Arbeitsplatzkultur führt das Institut Great Place to Work ${ }^{\circledR}$ seit 2002 regelmäßig Wettbewerbe wie „Deutschlands beste Arbeitgeber“ und „Europas beste Arbeitgeber“ oder Branchen- und Regionalwettbewerbe wie „Beste Arbeitgeber im Gesundheitswesen“ oder „Beste Arbeitgeber im Münsterland“ und „Beste Arbeitgeber in Berlin-Brandenburg“ durch. Ziel ist es, Unternehmen, die eine attraktive, mitarbeiterorientierte Arbeitsplatzkultur haben, besondere öffentliche Anerkennung zukommen zu lassen.

Alljährlich präsentiert das Institut mit dem Handelsblatt, dem Personalmagazin und dem Demographienetzwerk ddn die Gewinner des Wettbewerbs. Die Ergebnisse basieren auf Mitarbeiterbefragungen in den teilnehmenden Unternehmen zu zentralen Arbeitsplatzthemen wie Vertrauen in das Management, Qualität der Zusammenarbeit, Identifikation mit der Arbeit und dem Unternehmen insgesamt, berufliche Entwicklungsmöglichkeiten, Vergütung, Gesundheitsförderung und Work-Life-Balance. Darüber hinaus wurde die Qualität der Maßnahmen der Personal- und Führungsarbeit bewertet.

Für den Wettbewerb 2014 hatten sich bundesweit 580 Unternehmen aller Branchen, GröBen und Regionen beteiligt und einer unabhängigen Überprüfung gestellt.

Unternehmen Branche Mitarbeiter Homepage

\section{Top 3 der Unternehmen $\mathbf{5 0}$ bis $\mathbf{5 0 0}$ Mitarbeiter}

\begin{tabular}{lllcl}
\hline 1 & $\begin{array}{l}\text { Altenpflegeheim } \\
\text { St. Gereon }\end{array}$ & Gesundheit & 312 & $\begin{array}{l}\text { www.st-gereon. } \\
\text { info }\end{array}$ \\
\hline 2 & MaibornWolff GmbH & IT/Beratung & 110 & $\begin{array}{l}\text { www. } \\
\text { maibornwolff.de }\end{array}$ \\
\hline 3 & $\begin{array}{l}\text { Rewe Zweignieder- } \\
\text { lassung Hürth }\end{array}$ & Handel & 397 & www.rewe.de \\
\hline
\end{tabular}

Top 3 der Unternehmen 501 bis 2.000 Mitarbeiter

\begin{tabular}{lllrl}
\hline 1 & domino-world & Gesundheit & 535 & $\begin{array}{l}\text { www.domino- } \\
\text { world.de }\end{array}$ \\
\hline 2 & $\begin{array}{l}\text { W. L. Gore \& Associates } \\
\text { GmbH }\end{array}$ & Industrie & 1.586 & $\begin{array}{l}\text { www.gore.com/ } \\
\text { de_de/ }\end{array}$ \\
\hline 3 & $\begin{array}{l}\text { NetApp Deutschland } \\
\text { GmbH }\end{array}$ & IT & 648 & www.netapp.de \\
\hline
\end{tabular}




\begin{tabular}{|c|c|c|c|c|}
\hline & Unternehmen & Branche & Mitarbeiter & Homepage \\
\hline \multicolumn{5}{|c|}{ Top 3 der Unternehmen 2.001 bis 5.000 Mitarbeiter } \\
\hline 1 & Microsoft Deutschland & IT & 2.354 & www.microsoft.de \\
\hline 2 & IngDiba Frankfurt/Main & Finanzdienstleistung & 3.046 & www.ing-diba.de \\
\hline 3 & SICK AG & Elektronik & 2.998 & www.sick.com \\
\hline \multicolumn{5}{|c|}{ Top 3 der Unternehmen über 5.000 Mitarbeiter } \\
\hline 1 & $\begin{array}{l}\text { Volkswagen Financial- } \\
\text { Services }\end{array}$ & Finanzdienstleistungen & 5.239 & www.vwfsag.de \\
\hline 2 & $\begin{array}{l}\text { Techniker } \\
\text { Krankenkasse }\end{array}$ & Krankenkassen & 12.851 & www.tk.de \\
\hline 3 & Philips Deutschland & Elektrotechnik & 6.287 & www.philips.de \\
\hline
\end{tabular}

Quelle: Great Place to Work ${ }^{\circledR}$ Deutschland 2014

\section{Die 100 besten Arbeitgeber in Europa 2014}

Im Jahr 2003 zeichnete das Institut Great Place to Work ${ }^{\circledR}$ außerdem erstmals die „100 Besten Arbeitgeber Europas“ aus. Damit liegt 2014 zum 12. Mal das Ergebnis des Wettbewerbs „Europas Beste Arbeitgeber 2014“ vor. Ein erstes Ergebnis, so das Institut, lautet: „Europas beste Arbeitgeber werden immer besser und achten verstärkt auf die Bedürfnisse und Wünsche der einzelnen Mitarbeiter.“ Laut Institutsbericht „Working on the Human Touch“ zeigt sich, dass das „Vertrauen in die europäischen Arbeitgeber in den letzten fünf Jahren gewachsen ist. Damit verbreitet sich auch zunehmend eine mitarbeiterorientierte Arbeitsplatzkultur in Europa.“

Wie wird nun ein Unternehmen zu einem „European Player“? Um sich für den Wettbewerb zu qualifizieren, muss sich ein Unternehmen zuerst auf eine der landesweiten Beste-Listen platzieren. Das gelang 2013/2014 über 800 Unternehmen in 19 nationalen Listen. Diese Unternehmen wurden für den europaweiten Wettbewerb u. a. in die Größenklassen „Beste Große Arbeitgeber“ (Größenklasse mit mehr als 500 Mitarbeiter) und „Beste Kleine und Mittlere Arbeitgeber“ (Größenklasse mit 50 bis 500 Mitarbeiter) unterteilt.

\section{Die besten Arbeitgeber - Top Ten der KMU in Europa (50 bis 500 Mitarbeiter)}

\begin{tabular}{lllll} 
& Unternehmen & Branche & EU-Land & Homepage \\
\hline 1 & Cygni IT & Consulting & Schweden & www.cygni.se \\
\hline 2 & $\begin{array}{l}\text { Bofallesskaber- } \\
\text { ne Edelsvej }\end{array}$ & $\begin{array}{l}\text { Soziale Dienste und } \\
\text { stattliche Behörden }\end{array}$ & Dänemark & $\begin{array}{l}\text { www.edelsvej. } \\
\text { esbjergkommune. } \\
\text { dk }\end{array}$ \\
\hline 3 & Key Solutions & $\begin{array}{l}\text { Professional Services } \\
\text { Business Process Outsourcing } \\
\text { Call centers }\end{array}$ & Schweden & $\begin{array}{l}\text { www.keysolutions. } \\
\text { se }\end{array}$ \\
& & & \\
\hline
\end{tabular}




\begin{tabular}{lllll}
\hline 4 & $\begin{array}{l}\text { One Vision } \\
\text { Housing }\end{array}$ & Bau- und Immobilienwesen & UK & www.ovh.org.uk \\
\hline 5 & Hartmanns & Personal \& Personalbeschaffung & Dänemark & $\begin{array}{l}\text { www.hartmanns. } \\
\text { dk }\end{array}$ \\
\hline 6 & $\begin{array}{l}\text { Centiro } \\
\text { Solutions }\end{array}$ & $\begin{array}{l}\text { Information Technology } \\
\text { Software }\end{array}$ & Schweden & www.centiro.se \\
\hline 7 & Webstep & $\begin{array}{l}\text { Information Technology } \\
\text { IT Consulting }\end{array}$ & Norwegen & www.webstep.no \\
\hline 8 & Vincit & IT-Software & Finnland & www.vincit.fi \\
\hline 9 & Conscia & IT-Consulting & Dänemark & www.conscia.dk \\
\hline 10 & Fondia & Rechtsberatung & Finnland & www.fondia.fi \\
\hline
\end{tabular}

Quelle: Great Place to Work $®$ Institute, Inc., 2014

Die besten Arbeitgeber - Top Ten der Großunternehmen in Europa (ab 500 Mitarbeiter)

\begin{tabular}{lllll} 
& Unternehmen & Branche & EU-Land & Homepage \\
\hline 1 & Davidson & Consulting Engineering & Frankreich & www.davidson.fr \\
\hline 2 & $\begin{array}{l}\text { domino-world } \\
\text { TM }\end{array}$ & Gesundheit-Dienstleistungen & Deutschland & $\begin{array}{l}\text { www.domino- } \\
\text { world.de }\end{array}$ \\
\hline 3 & Torfs & Einzelhandel & Belgien & www.torfs.de \\
\hline 4 & $\begin{array}{l}\text { Capital One } \\
\text { (Europe) plc }\end{array}$ & Finanzdienstleistungen \& & UK & www.capitalone. \\
co.uk
\end{tabular}

Quelle: Great Place to Work ${ }^{\circledR}$ Institute, Inc., 2014 